\title{
Fatores associados à violência conjugal em um grupo de mulheres no município de João Pessoa - Paraíba. $^{1}$
}

\author{
Factors associated with domestic violence against \\ women in João Pessoa - Paraíba.
}

\section{Factores vinculados a la violencia conyugal en un grupo de mujeres en el municipio de João Pessoa - Paraíba.}

Jackeline Abílio de Souza²

Ana Tereza de Medeiros ${ }^{3}$

Ulisses Umbelino dos Anjos ${ }^{4}$

RESUMO: O estudo objetivou investigar fatores associados à violência conjugal em um grupo de mulheres no município de João Pessoa-PB. Para atender ao objetivo, realizou-se uma pesquisa do tipo analítico, com abordagem quantitativa, cuja ferramenta de análise estatística dos dados foi a Regressão Logística. O local do estudo correspondeu à Delegacia Especializada de Atendimento à Mulher do município de João Pessoa, onde foi selecionada, por conveniência, no período compreendido entre abril e agosto de 2012, uma amostra de 291 mulheres maiores de 18 anos, das quais 206 sofreram violência conjugal e 85 não sofreram violência conjugal a menos de um ano. As variáveis relacionadas às características pessoais, sociais e econômicas dos sujeitos do estudo foram identificadas no questionário aplicado às mulheres e utilizadas para a obtenção do modelo probabilístico final. Os resultados demonstraram que a situação da mulher ter sofrido violência anterior aumenta a chance de ser violentada novamente quase cinco vezes. Além disso, ser aposentada ou pensionista diminui a chance de a mulher sofrer agressão em quase nove vezes. No que tange aos homens, quando eles têm renda menor que dois salários mínimos e meio e têm história de violência na família, a chance de eles praticarem a violência contra a parceira

\footnotetext{
1 Trabalho extraído da dissertação "Fatores associados à violência conjugal no município de João Pessoa-PB, defendida junto ao Programa de Pós-Graduação em Modelos de Decisão e Saúde da Universidade Federal da Paraíba (UFPB). 2 Mestre em Modelos de Decisão e Saúde da UFPB. Doutoranda em Modelos de Decisão e Saúde da UFPB. Universidade Federal da Paraíba. Departamento de Enfermagem em Saúde Pública e Psiquiatria. Centro de Ciências da Saúde. Cidade Universitária - João Pessoa, PB. CEP: 58059-900. E-mail: jackabilio@yahoo.com.br 3 Doutora em Enfermagem. Docente do Departamento de Enfermagem em Saúde Pública e Psiquiatria da UFPB. Professora do Programa de Pós-graduação em Modelos de Decisão e Saúde do Centro de Ciências Exatas e da Natureza - CCEN da Universidade Federal da Paraíba-UFPB

4 Doutor em Estatística. Docente do Programa de Pós-Graduação em Modelos de Decisão e Saúde do Centro de Ciências Exatas e da Natureza - CCEN da Universidade Federal da Paraíba-UFPB
} 
aumenta em duas vezes. A chance de praticar a agressão diminui em quase duas vezes quando o homem é casado. Esses resultados indicam que a violência conjugal é um fenômeno complexo cuja ocorrência encontra-se associada a fatores de ordem pessoal, social, econômica e cultural. Descritores: Violência contra a Mulher, Causalidade, Identidade de Gênero,

\begin{abstract}
The aim of the present study was to investigate the factors associated with domestic violence against women in the city of João Pessoa, state of Paraíba, Brazil. For this purpose, we conducted a quantitative analytical study using logistic regression for the statistical analysis of the data. The study was conducted in the Police Station Specialized in Assistance to Women in João Pessoa, using a sample of 291 women older than 18 years of age, of which 206 had experienced domestic violence and 85 had not experienced domestic violence within one year of enrollment. The subjects were selected between April and August 2012 via convenience sampling. The variables related to personal, social, and economic characteristics were identified using a questionnaire and were used to construct the final probabilistic model. The results indicated that previous episodes of domestic violence against women involved an approximately five-fold increase in the likelihood of new episodes of violence. In addition, being retired or a pensioner involved an approximately nine-fold decrease in the chance of violence against women. With respect to men, an income lower than 2.5 times the minimum wage and a history of family violence involved a two-fold increase in the chance of abuse against the partner. The chance of aggression involved an approximately twofold decrease in cases in which men were married. These findings indicate that domestic violence is a complex phenomenon and that its occurrence is associated with personal, social, economic, and cultural factors.
\end{abstract}

Keywords: Violence against women, Causality, Gender Identity,

RESUMEN: El estudio tuvo como finalidad investigar factores vinculados a la violencia conyugal, en un grupo de mujeres en el municipio de João Pessoa/PB. Para alcanzar el objetivo, se realizó una investigación analítica, con abordaje cuantitativo, cuya herramienta de análisis estadístico de los datos fue la Regresión Logística. El estudio se llevó a cabo en la Comisaría Especializada de Atención a la Mujer de la ciudad de João Pessoa, donde se seleccionaron 291 mujeres mayores de 18 años, entre abril y agosto de 2012, de las cuales 206 sufrieron violencia conyugal y 85 no sufrieron violencia conyugal en el último año. Se identificaron las variables relacionadas con las características personales y socioeconómicas de los sujetos de estudio, que luego se utilizaron para obtener un modelo probabilístico final. Los resultados demostraron que el hecho de que la mujer haya sufrido violencia, en un período anterior, aumenta en cinco veces más la chance de sufrir nuevamente violencia. Además, ser jubilada o pensionista disminuye la chance de que la mujer sufra agresión en casi nueve veces. Respecto al hombre, cuando tiene una renta inferior a dos salarios mínimos y medio, y tiene un historial de violencia familiar, la chance de que él practique violencia contra su pareja aumenta el doble. La chance de practicar agresión disminuye en casi dos veces cuando el hombre es casado. A partir de los descubrimientos, se concluyó que la violencia 


\section{$83 / /$}

conyugal es un fenómeno complejo, cuya ocurrencia se encuentra vinculada a factores de orden personal, socioeconómico y cultural.

Descriptores: Violencia contra la mujer, Causalidad, Identidad de Género,

\section{INTRODUÇÃO}

Historicamente, as mulheres têm sido vitimizadas por diversas formas de discriminação e de desrespeito à dignidade humana através da limitação da sua participação na vida política, social, econômica e cultural dos diferentes países. Entre as formas mais atrozes de violação dos direitos humanos da mulher, a violência conjugal configura um dos tipos de agressão e é a que mais aflige o gênero feminino dentro do ambiente doméstico, espaço onde também são mantidas relações afetivas e compartilhados projetos de vida entre seus membros ${ }^{1}$.

No interior das relações conjugais, as mulheres expostas à violência chegam a sofrer cotidianamente diferentes tipos de agressões que incluem abuso sexual, físico, psicológico e moral, por meio de atos que podem ocasionar, desde lesões físicas e agudas, até agravos crônicos com repercussões na saúde mental e no bem-estar social ${ }^{2-3}$. Até o século XIX, os atos de violência conjugal cometidos contra a mulher, pelo seu companheiro, permaneceram silenciados no interior do espaço doméstico e somente nas últimas décadas têm sido visibilizados - principalmente por grupos feministas e por organizações internacionais - como um problema de magnitude mundial. No Brasil, o fenômeno também tem sido considerado como um problema da área da saúde pública, uma vez que impacta a saúde das mulheres e pode perturbar as suas potencialidades para contribuir para o desenvolvimento da sociedade, assim como, para a busca da autonomia ${ }^{2}$.

Motivados pelas amplas consequências - tanto individuais quanto coletivas - acarretadas pela violência, esse tema tem sido alvo de frequentes encontros internacionais que demandam a necessidade da ampliação do conhecimento sobre os fatores associados à ocorrência da violência, a fim de que os conhecimentos adquiridos subsidiem o planejamento e o desenvolvimento de ações para preveni-la e combatê-la de forma mais eficaz.

Nesse contexto, a Organização Pan Americana de Saúde recomendou que as pesquisas sobre a violência contribuam com o estudo de fatores que se associam a um maior ou menor risco da ocorrência do fenômeno, sobretudo fatores passíveis de mudanças provocadas por intervenções intersetoriais ${ }^{4}$. Vislumbra-se que esses estudos possam contribuir para a ampliação do conhecimento sobre a violência e possam fornecer dados que subsidiem estudos locais, nacionais e internacionais, especialmente voltados ao grupo das mulheres, tendo em vista que elas compõem um dos grupos populacionais mais vulneráveis a esse tipo de fenômeno ${ }^{5}$.

Diante da necessidade de pesquisas que abordem os fatores potencializadores da violência conjugal, este estudo pretendeu investigar os fatores que se associam à violência conjugal em um grupo de mulheres no município de João Pessoa-PB.

\section{MÉTODOS}

O estudo é do tipo analítico e observacional, com enfoque quantitativo. A análise estatística dos 
dados foi realizada mediante o emprego do modelo de regressão logística. A Regressão Logística corresponde a uma técnica utilizada para estudar a influência de fatores (variáveis) no aparecimento de um determinado fenômeno ou de uma característica através do estabelecimento de uma relação estatística entre o evento e as variáveis que caracterizam a população exposta ao fenômeno em estudo ${ }^{6}$.

Diferentemente de outros modelos de regressão, o modelo logístico é ideal nos casos em que a variável resposta (dependente) é qualitativa, ou seja, quando os dados são expressos na forma não numérica - por exemplo, sexo, cor de olhos, entre outros ${ }^{7,8}$. Isso possibilita estudar situações nas quais se pretende estimar a ausência ou a ocorrência de um evento (variável resposta) com base em um conjunto de fatores (variáveis explicativas) ${ }^{7,8}$. Uma vez que a variável resposta possui apenas dois grupos de interesse (resultados), que podem representar sucesso (caso o evento de interesse ocorra) ou fracasso (caso o evento de interesse não ocorra) ${ }^{9}$-, os valores da variável resposta y serão 0 ou 1 , conforme indica a seguinte demonstração ${ }^{10}$ :

\section{$\mathrm{y}=$}

Denomina-se "sucesso" "o resultado mais importante da resposta ou aquele que pretendemos relacionar com as demais variáveis de interesse" 11 . Desse modo, o grupo de interesse poderá ser codificado como 1 e o grupo de fracasso poderá ser codificado como 0 , ou vice-versa ${ }^{10,12}$. Nas situações em que $\mathrm{y}_{\mathrm{i}}$ assume apenas dois valores (0 ou 1), a distribuição da variável dependente é Bernoulli, um caso especial da distribuição binomial representada pela seguinte notação?:

$$
E(y)=\frac{\exp (x \beta)}{1+\exp (x \beta)} E(y)=\frac{\exp (x \beta)}{1+\exp (x \beta)}\left(\mathrm{y}_{\mathrm{i}} \mid \mathrm{x}_{\mathrm{i}}\right) \sim \operatorname{Bin}\left(\mathrm{n}_{\mathrm{i}}, \pi_{\mathrm{i}}\right) \quad \mathrm{i}=1,2 \ldots, \mathrm{n}
$$

Onde,

- $\quad \mathrm{y}_{\mathrm{i}}=\left[\mathrm{y}_{1}, \mathrm{y}_{2}, \ldots, \mathrm{y}_{\mathrm{n}}\right]$ é um vetor que contém os valores de $n$ observações da variável resposta ${ }^{10}$

- $\mathrm{n}_{\mathrm{i}}$ corresponde ao número de observações ou de valores de cada variável independente $\mathrm{x}_{\mathrm{i} 1}, \mathrm{x}_{\mathrm{i} 2}, \mathrm{x}_{\mathrm{i} 3}, \ldots, \mathrm{x}_{\mathrm{ip}}$;

- $\quad \mathrm{x}_{\mathrm{i}}=\left[1, \mathrm{x}_{\mathrm{i} 1}, \mathrm{x}_{\mathrm{i} 2}, \ldots, \mathrm{x}_{\mathrm{ip}}\right]^{\mathrm{T}}$ representa um vetor de valores de $p$ variáveis independentes ${ }^{10}$;

- $\pi_{\mathrm{i}}$ representa a probabilidade de sucesso da variável resposta dado $\mathrm{x}^{9,13}$.

De acordo com a demonstração anterior, o valor esperado da variável resposta, dado os valores das variáveis explicativas, equivale à probabilidade da variável y assumir o valor 1 , ou seja, $\mathrm{E}\left(\mathrm{y}_{\mathrm{i}} \mid\right.$ $\left.\mathrm{x}_{\mathrm{i}}\right)=\pi_{\mathrm{i}}$. Quando a variável reposta é binária, o valor do erro $€$ também assumirá apenas dois valores:

$\mathrm{O}$ valor do erro será $\epsilon=1-\pi_{\mathrm{i}}$, quando $\mathrm{y}_{\mathrm{i}}=1$

O valor do erro será $\epsilon=-\pi_{\mathrm{i}}$, quando $\mathrm{y}_{\mathrm{i}}=0$ 
A variância será dada por $\sigma_{\mathrm{yi}}^{2}=\pi_{\mathrm{i}}\left(1-\pi_{\mathrm{i}}\right)^{6,10,13}$. Considerando a natureza binária (do tipo Bernoulli) da variável dependente, a regressão logística prevê, a partir dos valores das variáveis independentes, valores de probabilidade que estejam contidos no intervalo entre 0 e 1 . Para garantir que os valores previstos para a variável resposta estejam compreendidos nesse intervalo, a função exponencial é empregada, resultando no seguinte modelo geral de regressão logística ${ }^{13}$ :

$$
\log \left\{\frac{\pi_{i}}{1-\pi_{i}}\right\}=\beta_{0}+\beta_{1} x_{1}+\ldots+\beta_{p} x_{p} \log \left\{\frac{\pi_{i}}{1-\pi_{i}}\right\}=\beta_{0}+\beta_{1} x_{1}+\ldots+\beta_{p} x_{p}
$$

Onde:

- $\beta=\left[\beta_{0}, \beta_{1}, \beta_{2}, \ldots, \beta_{\mathrm{p}}\right]^{\mathrm{T}}$ refere um vetor de parâmetros a ser estimado com base na variável dependente e nas variáveis independentes;

- $\quad \beta_{0}+\beta_{1} x_{1}+\ldots+\beta_{p} x_{p} \beta_{0}+\beta_{1} x_{1}+\ldots+\beta_{p} x_{p}$ representa a estrutura linear do modelo de regressão logística, denominada de preditor linear $\eta^{10}$;

- e $\log \left(\frac{\pi_{i}}{1-\pi_{i}}\right) \log \left(\frac{\pi_{i}}{1-\pi_{i}}\right)$, também chamado de função logit, representa o logaritmo da probabilidade do sucesso ocorrer $\left(\pi_{\mathrm{i}}\right)$ em relação à probabilidade do sucesso não ocorrer $\left(1-\pi_{\mathrm{i}}\right)^{9}$.

A relação $\pi_{1} / 1-\pi_{1}$ é denominada $o d d s$ e está condicionada ao incremento de uma unidade na variável explicativa $\mathrm{x}$. A razão condicionada à mudança de uma unidade na variável explicativa $\mathrm{x}$ é dennminada Odds Ratio n Razãn de Chance representada a seguir ${ }^{10}$ :

$$
\hat{O}_{R}=\frac{\pi_{1} / 1-\pi_{1}}{\pi_{0} / 1-\pi_{0}}=e^{\widehat{\beta_{j}}} \quad j=1, \ldots, \mathrm{p}
$$

Assim, a razão de chance é uma estimativa que traduz a influência da oscilação dos valores das variáveis explicativas no valor esperado da variável resposta, de modo que o acréscimo de uma unidade ao valor da variável preditora representa um acréscimo proporcional na probabilidade do sucesso ocorrer ${ }^{10}$. Assim, o $\hat{O}_{R} \hat{O}_{R}$, através do comportamento do coeficiente $\beta$, determina o quanto a $\pi_{\mathrm{i}}$ está aumentando ou diminuindo a cada unidade no valor de $\mathrm{x}^{14}$.

Note, na equação 1 , que a variável resposta $y_{i}$ não influencia a obtenção da $\pi_{i}$, pois a probabilidade do sucesso $\pi_{\mathrm{i}}$ depende apenas das variáveis preditoras e de um vetor de parâmetros $\beta$ desconhecidos ${ }^{9}$. Esses parâmetros estão relacionados à variável resposta y através da função logística ${ }^{12}$.

Considerando que a variável dependente assume apenas os valores 0 e 1, a razão de chance possibilita que o valor previsto para a variável dependente também assuma valores entre 0 e $1^{15}$. Desse modo, para cada observação, a técnica de regressão logística prevê um valor de probabilidade $\pi_{\mathrm{i}}$ entre 0 e 1 , gerando um gráfico de valores previstos - obtidos com base em todos os valores das variáveis independentes e dos parâmetros $\beta$ estimados $^{15}$.

A probabilidade prevista (valor esperado) é comparada a um ponto de corte (ex. 0,50), de modo que se ela for maior do que o ponto de corte pode-se prever que o resultado seja 1 (o evento ocorreu). Se a probabilidade prevista for menor do que o ponto de corte, pode-se prever que o resultado seja 0 (o evento não ocorreu) ${ }^{15}$. 
Tendo em vista que a variável resposta pertence à distribuição binomial, a relação entre o valor esperado $\mathrm{E}\left(\mathrm{y}_{\mathrm{i}} \mid \mathrm{x}_{\mathrm{i}}\right)$ e as variáveis explicativas do modelo é representada pela curva logística em forma de $\mathrm{S}^{15}$. No gráfico da curva logística, o eixo Y representa o valor esperado da variável resposta $\pi_{\mathrm{i}}$ e o eixo X representa o preditor linear $\eta$ - obtido com os valores das variáveis independentes e com os coeficientes $\beta$ estimados ${ }^{15}$.

Portanto, "com uma curva logística estimada, podemos estimar a probabilidade para qualquer observação com base em seus valores para as variáveis independentes e então prever a pertinência ao grupo usando um valor de corte" 15 . A curva logística demonstra que para valores muito baixos do preditor linear - dado por $\mathrm{x}_{\mathrm{i}}$ e os parâmetros $\beta$ estimados-, a probabilidade de o evento ocorrer se aproxima de 0; e quando os valores das variáveis independentes aumentam, os valores de $\mathrm{Y}$ crescem para cima da curva ${ }^{15}$. Essa relação entre a variável dependente (representada no eixo Y) e a independente (representada no eixo X) pode ser visualizada no gráfico por pontos dispersos na região superior e inferior da curva logística.

Os pontos resultantes das $\mathrm{n}_{\mathrm{i}}$ observações ficam dispersos próximos à curva logística, obedecendo a variabilidade da distribuição dos valores de y em relação a cada valor de $\mathrm{x}^{16}$. Dependendo da variabilidade das variáveis explicativas, a curva logística estimada se dispersa ou se aproxima do seu valor médio $\pi_{\mathrm{i}}$, decrescendo quando $\pi_{\mathrm{i}}$ tende a 0 e crescendo quando $\pi_{\mathrm{i}}$ tende a $1^{16}$.

A curva logística e os parâmetros $\beta_{0}, \beta_{1}, \ldots \beta_{\mathrm{n}}$ desconhecidos são estimados a partir dos dados amostrais de $\mathrm{y}_{\mathrm{i}}$ e de $\mathrm{x}_{\mathrm{i}}$, de modo a oferecer aos pontos $\left(\eta ; \pi_{\mathrm{i}}\right)$ a melhor aderência à curva logística real, resultando no melhor modelo para explicar a ocorrência de um fenômeno em investigação ${ }^{16}$. Devido à característica qualitativa da variável dependente e à utilização da transformação logit no Modelo de Regressão Logística, o método mais utilizado para a estimação dos parâmetros $\beta_{0}, \beta_{1}, \ldots \beta_{\mathrm{n}}$ é o da Máxima Verossimilhança, que estima o valor de $\beta$ maximizando a função de verossimilhança, demonstrada a seguir ${ }^{7,15}$ :

$$
L(\beta)=\prod_{i=1}^{n} \pi \mathrm{i}_{i}^{y i}\left(1-y_{i}\right)^{1-y i} L(\beta)=\prod_{i=1}^{n} \pi \mathrm{i}_{i}^{y i}\left(1-y_{i}\right)^{1-y i}
$$

Com base na função anteriormente apresentada, após algumas transformações é possível obter a seguinte função de máxima verossimilhança:

$$
\begin{aligned}
& L(\beta)=\sum_{i=1}^{n}\left[y_{i}\left(\beta_{0}+\beta_{1} x_{i}\right)-\ln \left(1+\exp \left(\left(\beta_{0}+\beta_{1} x_{i}\right)\right)\right)\right] \\
& L(\beta)=\sum_{i=1}^{n}\left[y_{i}\left(\beta_{0}+\beta_{1} x_{i}\right)-\ln \left(1+\exp \left(\left(\beta_{0}+\beta_{1} x_{i}\right)\right)\right)\right]
\end{aligned}
$$

A estimação dos parâmetros do modelo de Regressão logística requer a obtenção de um modelo cujas variáveis explicativas sejam significativas, ou seja, cujos parâmetros $\beta$ estimados sejam diferentes de zero $(\beta \neq 0)$. Entre as formas para averiguar a qualidade do ajuste do modelo, a função Desvio (Deviance) tem sido utilizada para comparar o modelo investigado ao melhor modelo possível (modelo saturado), baseada no logaritmo da função de verossimilhança ${ }^{7,11}$, como descreve a expressão a seguir:

$$
D=-2 \ln \left[\frac{\text { verossimilhança do modelo a justado }}{\text { verossimilhança do modelo saturado }}\right] D=-2 \ln \left[\frac{\text { verossimilhança do modelo a justado }}{\text { verossimilhança do modelo saturado }}\right]
$$

que resulta na seguinte estatística $D$ :

Tempus, actas de saúde colet, Brasília, 10(2), 81-102, jun, 2016. 


$$
D=-2 \sum_{i=1}^{n}\left\{y_{i} \log \left(\frac{\hat{\pi}_{i}}{y_{i}}\right)+\left(1-y_{i}\right) \log \left(\frac{1-\hat{\pi}_{i}}{1-y_{i}}\right)\right\} D=-2 \sum_{i=1}^{n}\left\{y_{i} \log \left(\frac{\hat{\pi}_{i}}{y_{i}}\right)+\left(1-y_{i}\right) \log \left(\frac{1-\hat{\pi}_{i}}{1-y_{i}}\right)\right\}
$$

O valor mínimo para a estatística $D$ é 0 , que corresponde a um ajuste perfeito, ou seja, ao melhor modelo possível ${ }^{15}$. O modelo nulo (modelo saturado) não contém variáveis independentes e é utilizado para ser comparado ao modelo proposto (investigado), que possui variáveis independentes a serem incluídas no modelo de regressão logística ${ }^{15}$. Para verificar a significância da diferença entre o melhor modelo possível e o modelo investigado são utilizados testes estatísticos, entre eles o teste qui-quadrado ${ }^{15}$.

Sob a hipótese nula de que os parâmetros estimados não apresentam relevância estatística $(\beta$ $=0)$ e da hipótese alternativa de que esses parâmetros são estatisticamente significantes $(\beta \neq 0)$, a estatística $D$ é comparada ao valor de Qui-quadrado $\left(X^{2}\right)$ - associado à um determinado nível de significância e à n-p graus de liberdade ${ }^{13}$. Se a hipótese nula não for aceita, o conjunto de variáveis independentes é significativo para a estimação do modelo, de modo que consegue explicar significativamente a ocorrência de um fenômeno em enfoque ${ }^{15}$.

\section{População e local do estudo}

O local do Estudo correspondeu à única Delegacia Especializada de Atendimento à Mulher - DEAM do município de João Pessoa-PB. A população compreendeu mulheres que estiveram presentes na DEAM no período destinado à coleta de dados - entre abril e agosto de 2012 -, sendo participantes: mulheres que sofreram violência conjugal recentemente (últimos 12 meses) e estiveram presentes na DEAM por motivo de denúncia da agressão; ou mulheres que estiveram na DEAM por outros motivos - como acompanhamento ou testemunho de algum conhecido - e que referiram não ter sofrido agressão por parceiro íntimo no último ano.

Portanto, foram formados dois grupos: um grupo de interesse - composto por mulheres que sofreram violência conjugal -; e outro grupo de mulheres que não tiveram episódio de violência conjugal há pelo menos um ano. As informações de ambos os grupos, levantadas através do instrumento de pesquisa, foram comparadas a fim de determinar os fatores que apresentaram associação à ocorrência da violência conjugal.

Considerando a dificuldade em conhecer o tamanho da população de mulheres que está exposta à violência no município de João Pessoa e ponderando que os dados oficiais podem estar subestimados - devido à ocultação da ocorrência da violência pelas mulheres vitimizadas -, o tamanho da amostra do estudo foi obtido por meio do dimensionamento amostral para população finita, representado pela seguinte equação:

$$
n=\frac{z_{G C, p \cdot q, N}^{2}}{\Theta^{2}(N-1)+z_{G C, p \cdot q}^{2}} n=\frac{z_{G C}^{2}, p \cdot q N}{\Theta^{2}(N-1)+z_{G C \cdot p \cdot q}^{2}}
$$

$\boldsymbol{p}$ corresponde à proporção amostral e $\boldsymbol{q}=1-p ; \boldsymbol{e}$ designa a margem de erro absoluta; e $\boldsymbol{Z}^{2}{ }_{\mathrm{GC}}$ representa um valor crítico ou escore $\mathrm{z}$ associado a um nível de confiança estabelecido pelo pesquisador (usualmente $90 \%, 95 \%$ ou $99 \%)^{8}$. O tamanho populacional $\mathrm{N}$ foi obtido neste estudo por meio de um levantamento prévio realizado pelos pesquisadores, quando foi verificada no ano 
de 2010 a ocorrência de 734 denúncias de casos de violência doméstica e familiar. O uso da fórmula com a estimativa $p$ motiva-se pela pretensão de estimar a proporção de mulheres em situação de violência.

Considerando $\boldsymbol{p}=\boldsymbol{q}=0,5$, um valor de erro de $5 \%$ e um nível de confiança de $95 \%$, ou seja, $\boldsymbol{\alpha}$ $=0,05$, obteve-se um valor de amostra de 252 indivíduos. Ao final do período designado para a coleta de dados, o número de mulheres entrevistadas alcançou a quantidade mínima de indivíduos requeridos para um tamanho de amostra com um erro de $5 \%$.

Considerando a dificuldade em extrair uma amostra de uma população que é reconhecida mais facilmente no momento em que a mulher denuncia a ocorrência da violência na DEAM, a seleção da amostra deste estudo foi por conveniência. No período designado para a coleta de dados (entre abril e agosto de 2012), as participantes foram selecionadas na DEAM no instante em que denunciavam a ocorrência da violência. Foram incluídas na amostra mulheres que tinham idade superior a 18 anos, que sofreram ou não qualquer tipo de agressão por indivíduo do sexo masculino com quem tenha mantido relações afetuosas (parceiro, ex-parceiro, cônjuge, ex-cônjuge, namorado ou exnamorado), que concordaram em participar do estudo e que assinaram o termo de consentimento livre e esclarecido.

\section{Instrumento de coleta de dados}

As informações foram levantadas através do instrumento de pesquisa (questionário). Os temas considerados no questionário foram abordados com base em depoimentos produzidos por uma pesquisa de enfoque qualitativo, a qual este estudo deu continuidade. O questionário aplicado continha 42 variáveis independentes, incluindo informações relativas às mulheres e aos cônjuges, entre elas: idade, estado civil, número de filhos, profissão, renda, religião, raça, escolaridade, tempo de relação com o cônjuge, convivência com situações de violência na família e na infância, violência anterior, uso de álcool e de drogas ilícitas.

\section{Procedimentos e Tratamento dos dados}

Após o período de coleta de dados, as informações extraídas através de questionário próprio foram revistas e transformadas em variáveis categóricas. As variáveis do estudo foram utilizadas para testar a hipótese nula $(\beta=0)$ de que fatores pessoais, sociais e econômicos não estão relacionados ao aumento da probabilidade de a mulher sofrer agressão pelo seu cônjuge.

Os dados foram digitados em uma planilha Excel, foram novamente checados quanto a possíveis erros ou inconsistências no preenchimento ou na digitação e foram categorizados conforme as tabelas 1 e 2 . A variável resposta foi codificada com apenas dois valores: com o valor 1 quando a violência conjugal ocorreu; e com valor 0 quando a violência conjugal não ocorreu. Com relação às variáveis explicativas, todas foram categorizadas - exceto as variáveis idade, tempo de relação e número de filhos (tabela 3).

As variáveis explicativas foram categorizadas como: raça/cor - branca, parda, preta e outras; estado civil - solteiro (a), casado (a), divorciado (a), viúvo (a) e separado (a); escolaridade - 
analfabeto(a) ou alfabetizado(a), ensino fundamental completo ou incompleto, ensino médio completo ou incompleto, ensino superior completo ou incompleto e pós-graduação; profissão desempregado(a), estudante, atividade remunerada e aposentado(a) ou pensionista; renda $-<1 / 2$ salário mínimo, $1 / 2$ a $1^{1 / 2}$ salário mínimo, $1^{1 / 2}$ a $2^{1 / 2}$ salários mínimos, $2^{1 / 2}$ a $3^{1 / 2}$ salários mínimos e $>3^{1 / 2}$ salários mínimos; religião - nenhuma, católica, evangélica, espírita e outras; uso de drogas e álcool - sim, não e não sabe; história de violência anterior - sim e não; história de violência na família e na infância - sim, não e não sabe.

Vale ressaltar que a categoria solteiro (a) incluiu mulheres ou homens em união estável não legalizada e a variável raça/cor foi autorreferida. Cada variável explicativa foi representada por uma abreviação, como mostram as Tabelas 1, 2 e 3 na sessão de resultados.

Após a fase de categorização das variáveis, foi iniciado o processo de análise estatística dos dados. Foi utilizada como ferramenta a Regressão Logística através do software The R Foundation for Statistical Computing (versão 2.13.0), de domínio público e acesso livre e gratuito. Essa ferramenta foi utilizada na conformação de um modelo estatístico contendo a variável resposta e variáveis explicativas estatisticamente significantes, conforme demonstrado nos resultados.

Tendo em vista o número de variáveis independentes - 23 ao todo - e as respectivas categorias ou fatores, foi realizada, inicialmente, uma análise logística simples entre a variável resposta e cada variável explicativa, a fim de testar individualmente a existência de relação significativa entre a variável testada e a variável de interesse. No ajuste do modelo de regressão simples, as variáveis cujo p.valor foi maior que $\alpha=0,05$ tiveram a hipótese nula não rejeitada, sendo, portanto, descartadas do modelo. As variáveis cujo p.valor foi menor que $\alpha=0,05$ tiveram a hipótese nula rejeitada e foram consideradas na predição do modelo.

Após definir as variáveis que comporiam o modelo inicial, denominado de modelo.1, os parâmetros das variáveis independentes selecionadas foram estimados conjuntamente e testados quanto à significância estatística. Após a estimação do modelo.1, foi realizado o seu ajuste por meio da seleção das variáveis explicativas significativas.

Durante o ajuste, as variáveis cujo p.valor foi menor que o nível de significância $\alpha=0,05$ tiveram a hipótese nula $(\beta=0)$ rejeitada e foram mantidas no modelo; as variáveis cujo p.valor foi maior que o nível de significância $\alpha=0,05$ tiveram a hipótese nula $(\beta=0)$ não rejeitada e foram excluídas do modelo. Sucessivamente, esses passos foram seguidos, de modo que a cada retirada de uma variável definia-se um novo modelo até o alcance do modelo final, chamado de Modelo.9 conforme o quadro 1.

No ajuste do modelo na análise multivariada, as variáveis tempo de relação conjugal (TEM), renda da mulher (RENM), escolaridade do homem (ESCH), número de filhos do homem (FILH), raça da mulher (RACM), violência na infância do homem (INF), religião do homem (RELH) e profissão do homem $(\mathrm{PROH})$ foram sequencialmente eliminadas. Após a seleção das variáveis, obteve-se o modelo final contendo 5 (cinco) variáveis preditoras que, conjuntamente, tinham relação significativa com a variável resposta. 
Quadro 1 - Processo de Ajuste do Modelo de Regressão Logística e de obtenção do modelo final

\begin{tabular}{|c|c|c|c|c|c|c|c|c|}
\hline \multicolumn{9}{|c|}{ Ajuste do Modelo de Regressão Logística } \\
\hline 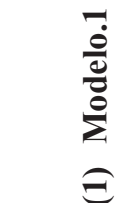 & 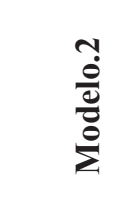 & 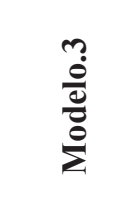 & $\begin{array}{l}\frac{\pi}{0} \\
\frac{0}{\pi} \\
\frac{\delta}{\delta}\end{array}$ & 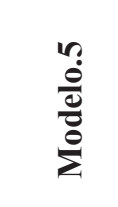 & $\begin{array}{l}0 \\
\dot{0} \\
\frac{0}{0} \\
\dot{0} \\
\dot{\Sigma}\end{array}$ & $\frac{\hat{0}}{\frac{0}{\tilde{g}}}$ & $\begin{array}{l}\infty \\
\dot{0} \\
\dot{0} \\
\dot{0} \\
\dot{0}\end{array}$ & 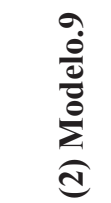 \\
\hline TEM & - & 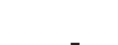 & 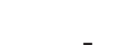 & 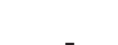 & - & - & 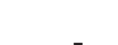 & - \\
\hline RENM & RENM & & & & & & & - \\
\hline $\mathrm{ESCH}$ & ESCH & ESCH & - & - & - & - & ـ & - \\
\hline FILH & FILH & FILH & FILH & _ & - & _ & - & - \\
\hline RACM & RACM & RACM & $\mathrm{RACM}$ & RACM & & & & - \\
\hline INF & INF & INF & INF & INF & INF & & & - \\
\hline RELH & RELH & RELH & RELH & RELH & RELH & RELH & - & - \\
\hline PROH & PROH & PROH & $\mathrm{PROH}$ & $\mathrm{PROH}$ & $\mathrm{PROH}$ & $\mathrm{PROH}$ & PROH & _ \\
\hline PROM & PROM & PROM & PROM & PROM & PROM & PROM & PROM & PROM \\
\hline ANT & ANT & ANT & ANT & ANT & ANT & ANT & ANT & ANT \\
\hline ESTH & ESTH & ESTH & ESTH & ESTH & ESTH & ESTH & ESTH & ESTH \\
\hline RENH & RENH & RENH & RENH & RENH & RENH & RENH & RENH & RENH \\
\hline FAMH & FAMH & FAMH & FAMH & FAMH & FAMH & FAMH & FAMH & FAMH \\
\hline
\end{tabular}

(1) Modelo inicial contendo as variáveis independentes - relacionadas aos sexos feminino e masculino - que foram estatisticamente significantes na análise logística simples. (2) Modelo final ajustado contendo as variáveis independentes - relacionadas aos sexos feminino e masculino - que melhor explicaram a ocorrência da violência conjugal.

Após definir o modelo de regressão logística (modelo.9), a qualidade do ajuste foi verificada utilizando o Teste de Adequação Global do Modelo através da função desvio e da estatística $D$ (Deviance). O teste possibilitou comparar o modelo proposto (modelo.9) a um modelo ideal, a fim de verificar se a aproximação entre eles era significativa o suficiente para aceitá-lo como adequado para explicar a ocorrência da variável resposta com base em um conjunto de variáveis explicativas selecionadas. Para tanto, a medida deviance, dividida pelo parâmetro de dispersão do modelo, foi comparada ao valor Qui-quadrado $\left(X^{2}\right)$ - a um nível de 95\% de confiança.

Sob a hipótese nula de que os parâmetros das variáveis adotadas não tinham relevância estatística, o teste demonstrou que o valor do desvio $(=290.0449)$ foi menor que o valor quiquadrado (=325.3741), o que acarretou a não aceitação da hipótese nula e a confirmação de que o conjunto das variáveis independentes do modelo proposto consegue explicar a ocorrência da violência conjugal em um grupo de mulheres no município de João Pessoa-PB.

Com base nos coeficientes $\beta$ estimados no modelo final, foi obtida a razão de chance para cada 


\section{$91 / /$}

variável independente. A Odds Ratio (OR), ou Razão de Chance, atribuiu valores que indicaram a influência da oscilação dos valores das variáveis explicativas no valor esperado da variável resposta, conforme apresentado nos resultados. Desse modo, baseado no cálculo do OR foi possível verificar a associação entre fatores sociais, econômicos e pessoais à violência conjugal.

\section{Aspectos Éticos da Pesquisa}

Vale salientar que este estudo buscou atender às diretrizes e às normas regulamentadoras de pesquisas envolvendo seres humanos, aprovadas pelo Conselho Nacional de Saúde e dispostas na Resolução n ${ }^{\circ} 466$ de 12 de dezembro de 2012, respeitando os cinco referenciais básicos da bioética autonomia, não maleficência, beneficência, justiça e equidade ${ }^{17}$. O artigo foi extraído da dissertação "Fatores associados à violência conjugal no município de João Pessoa-PB" cuja autora foi bolsista pela Coordenação de Aperfeiçoamento de Pessoal de Nível Superior/CAPES. A pesquisa trata-se da continuidade de um estudo qualitativo, apresentado como Trabalho de Conclusão de Curso em 2010, cujo projeto foi aprovado pelo Comitê de Ética em Pesquisa Envolvendo Seres Humanos do Hospital Universitário Lauro Wanderley - H.U.L.W. (protocolo 431/09).

\section{RESULTADOS}

Neste estudo, foram realizadas 325 entrevistas, das quais 34 foram eliminadas por motivo de desistência das participantes durante a entrevista ou porque elas forneceram informações incompletas. Entre as 291 pessoas entrevistadas, 206 corresponderam às mulheres que sofreram violência conjugal e 85 compreenderam mulheres sem episódio de violência pelo parceiro a menos de um ano.

A análise da regressão logística simples entre a variável resposta e cada uma das 23 variáveis explicativas demonstrou significância estatística (p.valor $<0,05$ ) em treze variáveis preditoras, das quais cinco relacionadas à mulher - raça, profissão, renda, tempo de relação conjugal e violência anterior (Tabelas 1 e 3) - e oito relacionadas ao homem - estado civil, escolaridade, profissão, renda, número de filhos, religião e história de violência na família e na infância (Tabelas 2 e 3 ).

$\mathrm{Na}$ análise multivariada, após o ajuste do modelo logístico obteve-se um modelo final contendo apenas cinco variáveis preditoras, entre elas estão as relacionadas às mulheres - profissão e existência de violência anterior - e as referentes aos homens - estado civil, renda e ocorrência de violência na família. $\mathrm{O}$ ajuste do Modelo final demonstrou que a característica feminina de ser aposentada ou pensionista $(\mathrm{p}=0,00593)$ representou fator de proteção contra a violência. Por outro lado, a característica de ter sofrido violência anterior $(\mathrm{p}=8,11 \mathrm{e}-08)$ foi reconhecida como fator de risco para a mulher sofrer qualquer tipo de agressão.

Quanto aos homens, a característica de ser casado $(\mathrm{p}=0,02313)$ representou fator de proteção para as mulheres contra os atos violentos. $\mathrm{O}$ fato de o homem receber renda menor que dois salários mínimos e meio $(\mathrm{p}=0,02332)$ ou ter história de violência na família $(\mathrm{p}=0,02264)$ representou fator de risco para praticar agressão contra sua parceira. Portanto, o ajuste do modelo de regressão logística multivariada permitiu identificar variáveis - do contexto social, econômico e pessoal de mulheres e de homens - que se associam à maior probabilidade da ocorrência da violência conjugal. 
Com relação às características masculinas, observou-se a redução em quase duas vezes a chance de praticar a agressão contra a parceira quando o homem saía da situação civil solteiro, viúvo ou divorciado para casado. Quando ele recebia uma renda menor que dois salários mínimos e meio ou tinha história de violência na família, essas características aumentaram 2,02 e 2,06 vezes, respectivamente, a chance de ele praticar a violência conjugal.

Tabela 1 - Valores dos coeficientes, do desvio padrão e do p.valor das variáveis explicativas categóricas relacionadas ao sexo feminino após análise logística simples

\begin{tabular}{|c|c|c|c|c|}
\hline *Variáveis/categorias & & $\begin{array}{c}\text { Categorias após o ajuste } \\
\text { do modelo logístico } \\
\text { multivariado }\end{array}$ & $\begin{array}{l}\text { Coeficientes } \\
\text { (Desvio } \\
\text { padrão) }\end{array}$ & P. valor \\
\hline \multicolumn{5}{|l|}{$\underset{1}{\operatorname{Racca}(\text { RACM) }}$} \\
\hline 2 Parda & 1 & Parda ou Preta & 0.5744 & $* *$ \\
\hline 3 Preta & 0 & Branca ou outras raças & $(0.2649)$ & 0.030 \\
\hline 4 Indígena & & & & \\
\hline \multicolumn{5}{|l|}{ Estado civil (ESTM) } \\
\hline $1 \quad$ Solteira & & & & \multirow{5}{*}{0.090} \\
\hline 2 Casada & & & & \\
\hline 3 Divorciada & $\begin{array}{l}0 \\
0\end{array}$ & Nãocacada & $(0.4836)$ & \\
\hline 4 Viúva & 0 & Não casada & & \\
\hline 5 Separada & & & & \\
\hline \multicolumn{5}{|l|}{ Escolaridade (ESCM) } \\
\hline 1 E. Superior Completo/Incompleto & 1 & E. Médio completo ou & & \multirow{4}{*}{0.254} \\
\hline 2 E. Médio Completo/Incompleto & & incompleto ou E. Superior & & \\
\hline 3 E. Fundamental Completo/Incompleto & & Completo & $(0.2786)$ & \\
\hline 4 Analfabeto ou Alfabetizado & 0 & Outros & & \\
\hline \multicolumn{5}{|l|}{ Profissão (PROM) } \\
\hline 1 Desempregado & & & & \multirow{4}{*}{$* * 0.010$} \\
\hline 2 Estudante & 1 & Aposentada ou pensionista & -1.8038 & \\
\hline 3 Atividade remunerada & 0 & Outros & $(0.7028)$ & \\
\hline $4 \quad$ Aposentado/Pensionista & & & & \\
\hline \multicolumn{5}{|l|}{ Renda (RENM) } \\
\hline $1<1 / 2$ salário mínimo & & & & \multirow{5}{*}{$\begin{array}{r}* * * \\
0.010\end{array}$} \\
\hline $2 \quad 1 / 2$ a $1^{1 / 2}$ salários mínimos & 1 & $<2^{1 / 2}$ salários mínimos & & \\
\hline $3 \quad 1^{1 / 2}$ a $2^{1 / 2}$ salários mínimos & $\begin{array}{l}1 \\
0\end{array}$ & $>2^{1 / 2}$ salárics mínimes & 0.1343 & \\
\hline $4 \quad 2^{1 / 2}$ a $3^{1 / 2}$ salários mínimos & & & & \\
\hline $5>3^{1 / 2}$ salários mínimos & & & & \\
\hline \multicolumn{5}{|l|}{ Religião (RELM) } \\
\hline 1 Católica & & & & \multirow{4}{*}{0.092} \\
\hline 2 Evangélica & 1 & Evangélica & -1.1494 & \\
\hline 3 Nenhuma & 0 & Outras Religiões & $(0.6837)$ & \\
\hline 4 Outras & & & & \\
\hline \multicolumn{5}{|l|}{ Violência na família (FAM) } \\
\hline 1 Presenciou violência na família & 1 & Violência na família & 0.001848 & \multirow{3}{*}{0.994} \\
\hline 2 Não presenciou violência na família & 0 & Violência não presente & $(0.259295)$ & \\
\hline Violência anterior (ANT) & & & & \\
\hline 1 Sofreu violência anteriormente & 1 & Violência anterior & 1.6409 & $* *$ \\
\hline 2 Não sofreu violência anteriormente & 0 & Violência não presente & $(0.2785)$ & $3.83 \mathrm{e}-09$ \\
\hline
\end{tabular}

Nota: $\mathrm{Na}$ análise logística simples, as categorias relevantes foram codificadas com o valor $1 \mathrm{e}$ as demais com o valor 0 . *Variáveis submetidas à análise logística simples. **Categorias estatisticamente significantes após análise logística simples com cada uma das variáveis relacionadas ao sexo feminino.

Tempus, actas de saúde colet, Brasília, 10(2), 81-102, jun, 2016. 
Tabela 2 - Valores dos coeficientes, do desvio padrão e do p.valor das variáveis explicativas categóricas relacionadas ao sexo masculino após análise logística simples

\section{*Variáveis/categorias}

Estado civil (ESTH)

$\begin{array}{ll}1 & \text { Solteiro } \\ 2 & \text { Casado } \\ 34 & \text { Divorciado } \\ 5 & \text { Viúvo } \\ & \text { Separado }\end{array}$

Escolaridade $(\mathrm{ESCH})$

1 E. Superior completo/ incompleto

2 E. Médio completo/incompleto

3 E. Fundamental completo/incompleto

4 Analfabeto ou Alfabetizado

5 Pós-graduação ou não sabe

Profissão (PROH)

$\begin{array}{ll}1 & \text { Desempregado } \\ 2 & \text { Estudante } \\ 3 & \text { Atividade remunerada } \\ 4 & \text { Aposentado/Pensionista }\end{array}$

Renda (RENH)

$\begin{array}{ll}1 & <1 / 2 \text { salário mínimo } \\ 2 & 1 / 2 \text { a } 1^{1 / 2} \text { salários mínimos } \\ 3 & 1^{1 / 2} \text { a } 2^{1 / 2} \text { salários mínimos } \\ 4 & 2^{1 / 2} \text { a } 3^{1 / 2} \text { salários mínimos } \\ 5 & >3^{1 / 2} \text { salários mínimos }\end{array}$

Religião (RELH)

1 Católica

2 Evangélica

3 Nenhuma

4 Outras

Uso de drogas (DROGA)

1 Faz uso de drogas ilícitas

2 Não faz uso de drogas ilícitas

3 Não Sabe

Uso de álcool (ALCOOL)

1 Faz uso de álcool

2 Não faz uso de álcool

Violência na família (FAMH)

1 Presenciou violência na família

2 Não presenciou violência na família

3 Não sabe

Violência na infância (INF)

1 Sofreu violência na infância

2 Não sofreu violência

3 Não sabe

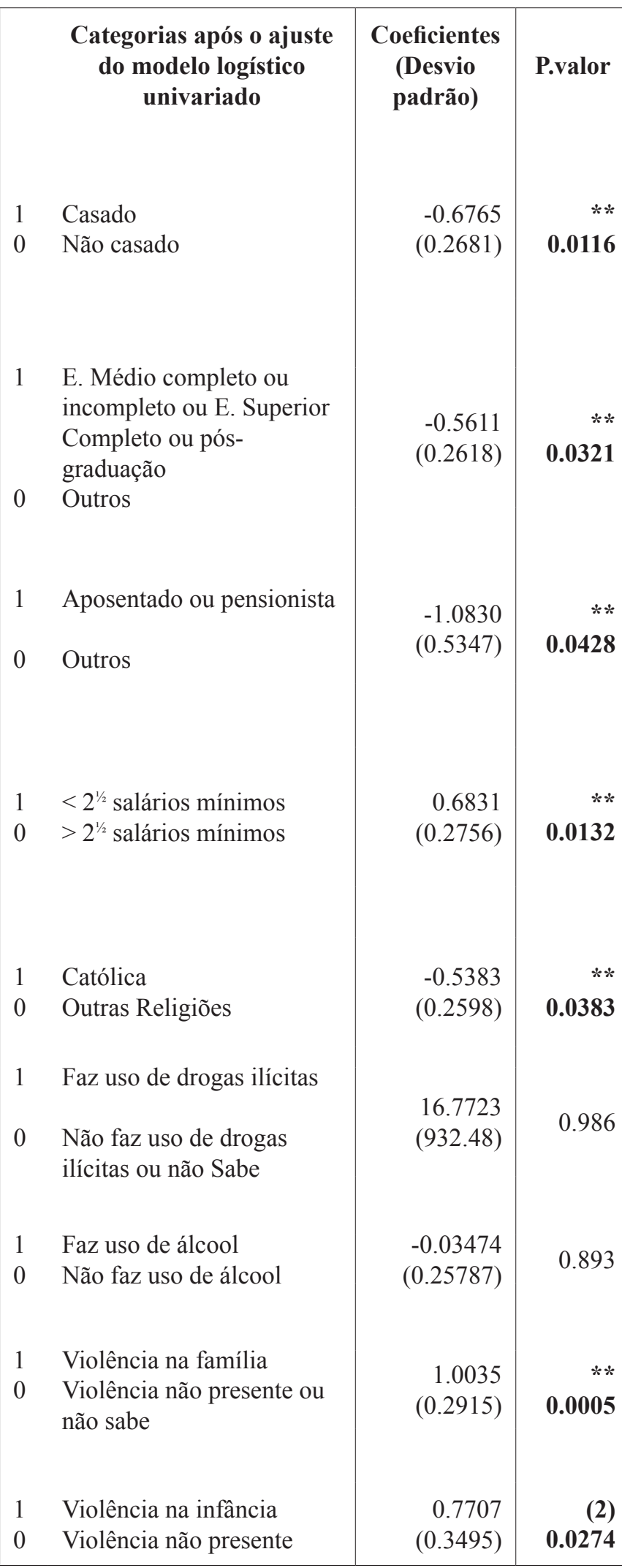

Nota: Na análise logística simples, as categorias relevantes foram codificadas com o valor 1 e as demais com o valor $0 .{ }^{*}$ Variáveis submetidas à análise logística simples. **Categorias estatisticamente significantes após análise logística simples com cada uma das variáveis relacionadas ao sexo masculino. 
Tabela 3 - Valores dos coeficientes, do desvio padrão e do p.valor das variáveis explicativas contínuas referentes aos sexos feminino e masculino após a análise logística simples

\begin{tabular}{l|c|c}
\hline \multicolumn{1}{c|}{ Variáveis Contínuas } & Coeficientes (Desvio padrão) & P-valor \\
\hline Idade da Mulher (IDM) & $-0.02295(0.01181)$ & 0.051915 \\
Número de Filhos da mulher (FILM) & $-0.2922(0.2854)$ & 0.306 \\
Tempo de relação (em anos) do casal (TEM) & $-1.0222(0.3710)$ & $* \mathbf{0 . 0 0 5 8 6}$ \\
Idade do Homem (IDH) & $-0.0183(0.0103)$ & 0.0773 \\
Número de Filhos do homem (FILH) & $-0.7116(0.3430)$ & $* \mathbf{0 . 0 3 8}$ \\
Número de Filhos extraconjugais do homem (FILH2) & $0.05376(0.07114)$ & 0.45 \\
\hline
\end{tabular}

*Variáveis contínuas estatisticamente significantes após a análise logística simples com cada uma das variáveis independentes referentes aos sexos feminino e masculino.

Tabela 4 - Modelo Final e Odds Ration (OR) das variáveis independentes resultantes da análise de regressão logística multivariada

\begin{tabular}{l|ccccc}
\hline \multicolumn{1}{c|}{ Modelo Final } & Coeficientes & Std.Error & P.Valor & OR & IC95\% \\
\hline $\begin{array}{l}\text { *Profissão: } \\
\text { Aposentada ou pensionista }\end{array}$ & -2.1969 & 0.7985 & 0.00593 & 0.1111464 & $0.023-0.531$ \\
*Violência anterior & 1.6060 & 0.2994 & $8.11 \mathrm{e}-08$ & 4.9829605 & $2.771-8,960$ \\
$\begin{array}{l}\text { **Estado civil: } \\
\begin{array}{l}\text { Casado } \\
\text { **Renda: }\end{array}\end{array}$ & -0.6857 & 0.3019 & 0.02313 & 0.5037429 & $0.279-0.910$ \\
$\begin{array}{l}<2^{1 / 2} \text { salários mínimos } \\
\text { **História de violência na } \\
\text { família }\end{array}$ & 0.7047 & 0.3107 & 0.02332 & 2.0231556 & $1.100-3.719$ \\
\hline
\end{tabular}

*Variáveis independentes relacionadas ao sexo feminino. **Variáveis independentes relacionadas ao sexo masculino. IC95\%: intervalo de 95\% de confiança. OR: Odds Ratio.

A definição do modelo final possibilitou obter os valores do Odds Ratio (OR) que indicaram a chance de as variáveis independentes aumentarem ou diminuírem a probabilidade da violência conjugal ocorrer, como demonstrado na tabela 4. Conforme os resultados, quando a mulher não estava na situação de desempregada ou de estudante e não estava exercendo qualquer atividade remunerada, mas estava aposentada ou recebendo pensão, a chance de sofrer violência pelo parceiro diminuiu quase nove vezes. Quando ela tinha história de violência anterior, a chance de sofrer a violência novamente aumentou 4,98 vezes. 


\section{DISCUSSÃO}

A análise logística multivariada revelou que as características que melhor explicaram a ocorrência da violência conjugal no grupo de mulheres estudado foram: a profissão e a história de violência anterior, além dos aspectos relacionados ao grupo masculino como estado civil, renda e convivência com situações de violência familiar.

Conforme demonstram os resultados, $71,35 \%$ da população feminina, vítima de agressão pelo cônjuge, exercia alguma atividade remunerada no momento da entrevista e apenas 1,45\% das mulheres eram aposentadas ou pensionistas. Apesar de aquelas mulheres receberem algum rendimento, cerca de $90 \%$ das que foram violentadas recebiam menos que dois salários mínimos e meio. Nesse contexto, a análise estatística demonstrou que ser aposentada ou pensionista - quando comparada às outras situações como ser desempregada ou estudante ou exercer qualquer atividade remunerada - diminuiu a chance de a mulher sofrer violência conjugal, possivelmente devido a esse tipo de ocupação proporcionar uma maior estabilidade financeira para o grupo feminino.

Apesar de não estar contida no modelo final, a renda também demonstrou ser significativa para a ocorrência da agressão quando as mulheres recebiam remuneração de até dois salários mínimos e meio. Esses resultados foram semelhantes aos apresentados em uma pesquisa brasileira realizada no Estado do Espírito Santo, na qual a maior parte das vítimas de agressão referia receber renda entre 1 a 3 salários mínimos ${ }^{18}$, situação semelhante também em outros estudos ${ }^{19,20}$. Entre as ocupações de baixa remuneração há empregadas domésticas, copeiras e faxineiras ${ }^{20,21}$.

Com relação às condições socioeconômicas, uma pesquisa realizada na Alemanha referiu uma tendência à ocorrência de agressão física praticada pelo parceiro íntimo contra mulheres que convivem com situações de pobreza, com um baixo nível socioeconômico e com uma baixa renda ${ }^{22}$. Naquele contexto, a dependência econômica feminina do parceiro, somada ao número de filhos e à falta de apoio social foram consideradas responsáveis pela elevação da vulnerabilidade da mulher para sofrer violência, podendo dificultar a libertação da opressão ${ }^{19,23}$.

O grau de instrução também tem sido apontado em diversas pesquisas como um fator predisponente à ocorrência da violência contra a mulher no espaço doméstico, principalmente quando o nível de instrução configura entre os mais baixos ${ }^{19,24}$. Essa situação foi observada em estudo realizado no Rio de Janeiro ao revelar que a maioria das mulheres violentadas que prestou queixa na delegacia especializada entre os anos de 2003 e 2008 apresentava apenas o ensino fundamental completo ${ }^{21}$. Em outro estudo realizado no estado do Rio Grande do Sul, observou-se que entre as mulheres que prestaram queixa por agressão na Delegacia Especializada, 63,2\% não chegaram a cursar o ensino médio $^{25}$.

No que diz respeito à figura masculina, foi demonstrada relação estatística negativa entre a prática da agressão contra a parceira e o grau de escolaridade acima do ensino médio incompleto. 
Receber uma renda de até dois salários mínimos e meio aumentou duas vezes a chance de o cônjuge agredir a mulher. Também foi demonstrado que 57,1\% dos autores da violência conjugal exerciam algum tipo de ocupação, no entanto, $71,4 \%$ tinham cursado até o ensino fundamental completo ${ }^{18}$.

A pobreza é um importante fator desencadeante de conflitos familiares que podem culminar com a violência contra as mulheres dentro do lar. A fome, as privações de lazer, a educação e a baixa qualidade de vida são condições que acarretam instabilidade emocional e afetiva entre os membros da família, criando um meio propício ao desenvolvimento de conflitos entre o marido e a parceira ${ }^{25}$. Os desentendimentos podem ser gerados devido à cobrança cultural de provisão econômica conferida aos homens e à necessidade de as mulheres complementarem e, muitas vezes, dominarem a provisão econômica da família ${ }^{23}$.

A convivência com a pobreza potencializa em alguns homens o surgimento de estresse, de frustração e de um sentimento de inadequação por não poderem cumprir com o papel de provedor culturalmente definido, situação que pode desencadear a violência ${ }^{27}$. Tradicionalmente, na construção da identidade de gênero, atribuiu-se à figura masculina o papel de provedor da família e às mulheres a dependência econômica dos homens. A inversão desses papéis configura-se uma transgressão dos padrões tradicionais de gênero e, em decorrência, em locais onde esses padrões ainda não estão adaptados à nova realidade de liberdade econômica feminina, principalmente em regiões rurais, a maior autonomia financeira da mulher é considerada fator de risco para sofrer violência ${ }^{23}$. Por isso, a "entrada da mulher no mercado de trabalho" também representa um fator que "desestabiliza o relacionamento conjugal baseado em diferenças"28.

Alguns autores explicam que "em crises ou ameaças de ruptura dessa dominação tradicional, comportamentos de reconquista do poder e da autoridade perdidos ou simplesmente de prevenção dessa perda serão não só possíveis, no universo simbólico da masculinidade hegemônica, mas necessários" 29. Já em contextos onde a independência econômica é característica de valorização feminina, o seu alcance parece ser um importante caminho para a prevenção e para o combate à violência contra a mulher ${ }^{23}$.

Considerando que os atos infringidos contra os indivíduos podem ser reconhecidos como violência com base em nuances diferenciadas pelo âmbito cultural, a convivência familiar com a presença de violência em algum momento da formação das identidades masculinas e femininas pode aumentar o risco de a mulher sofrer agressões. Desse modo, homens, que durante sua infância não tiveram boa relação com seus pais, conviveram com situações de violência (tanto por presenciar a sua ocorrência, quanto por sofrer agressões por parte de seus pais ou figuras paternas) e viveram em um ambiente marcado pela falta de diálogo, autoritarismo paterno e submissão materna, podem reproduzir a violência nas suas relações sociais e no casamento contra suas parceiras ${ }^{30}$.

Sobre esse assunto, uma investigação com a participação de 790 mulheres que foram agredidas pelo parceiro íntimo apontou que a convivência com a violência esteve fortemente associada a 
problemas de comportamento dos filhos ${ }^{31}$. Por outro lado, a harmonia familiar parece ser um fator protetor contra o comportamento antissocial em lares onde há boas relações entre os pais (ou outros adultos) e os filhos ${ }^{32}$.

A característica de ter presenciado pelo menos um evento de violência entre membros da família foi identificada como um fator que aumenta a chance de o homem agredir sua companheira. Uma investigação demonstrou ser positiva a associação entre a ocorrência da violência cometida contra a mulher e a existência de violência na família do autor da agressão ${ }^{21}$. Quando analisada a história de violência familiar em mulheres que sofreram agressão, também revelou-se ser significativa a associação entre a ocorrência da violência física e a situação de terem presenciado agressão cometida contra as suas mães por parceiro íntimo ${ }^{31}$.

O fato de a mulher ter sofrido violência anterior cometida por qualquer pessoa do seu contexto doméstico também foi revelado como fator significativo para ela sofrer a violência conjugal atual. A maior parte das mulheres que sofre agressão e denuncia a ocorrência na delegacia especializada de atendimento revela ter sido agredida anteriormente ${ }^{18,34}$. Mulheres que sofreram violência física em algum momento da sua vida têm $29 \%$ mais chance de serem abusadas sexualmente por seus parceiros do que as que não conviveram com esse abuso ${ }^{30}$.

A recorrência das agressões acarreta reincidências das denúncias realizadas pelas mulheres nas delegacias especializadas em uma proporção de $25,2 \%$ dos registros ${ }^{25}$. Um dos motivos para as mulheres permanecerem nas relações conjugais com a reincidência de violência têm sido, na maioria dos casos investigados, "o desejo de manter a união familiar na companhia dos filhos". ${ }^{35}$

Desse modo, os resultados deste estudo - respaldados por outras pesquisas nacionais e internacionais - revelam a existência de uma multiplicidade de fatores pessoais, sociais, econômicos, familiares e vivenciais que, no processo de formação das identidades de gênero, podem favorecer a ocorrência da violência no espaço doméstico $22-23,26,36-38$.

A natureza dos fatores estudados demonstrou a influência de não apenas condições individuais, familiares e comunitárias na ocorrência da violência conjugal, mas apontou também a determinação do fenômeno por condições geradas em níveis de influência superiores e indiretos, que podem interferir na dinâmica da vida diária dos indivíduos. Um desses fatores é a existência de políticas que mantêm as desigualdades socioeconômicas e as desigualdades de gênero ${ }^{32}$.

No entanto, apesar de os resultados encontrados neste estudo, eles não podem ser generalizados para a população, uma vez que a seleção da amostra foi obtida por meio de um método não probabilístico. A escolha de um método não probabilístico esteve relacionada à dificuldade de acessibilidade aos elementos da população, tendo em vista que a população de mulheres que sofre ou sofreu violência conjugal é reconhecida mais facilmente no momento em que elas denunciam a ocorrência das agressões na DEAM. Além disso, considerou-se a indisponibilidade de tempo e 
de recursos financeiros e humanos que seriam demandados para extrair uma amostra probabilística satisfatória.

\section{CONSIDERAÇÕES FINAIS}

O estudo apontou que a situação ocupacional - como baixos níveis de remuneração - e a situação de conviver ou sofrer com a violência na infância ou na família oferecem risco para a mulher ser violentada pelo cônjuge. Ao considerar esses fatores, reitera-se que a compreensão da violência conjugal deve ser pautada numa abordagem multifatorial, com base no reconhecimento do problema como um evento que resulta da influência de aspectos de ordem pessoal, social, econômica e cultural de mulheres e de homens.

Ao reiterar que situações individuais, interpessoais, comunitárias e sociais podem determinar atos violentos por parte de homens contra as suas parceiras, entende-se que, assim como afirmam organismos nacionais e internacionais, sozinho o setor da saúde não conseguirá apresentar respostas significativas no que diz respeito ao combate à violência. Esse setor necessita, portanto, ser inserido num amplo projeto intersetorial em busca de um objetivo comum - reduzir, controlar e prevenir a violência.

$\mathrm{Na}$ área da saúde, especialmente no Brasil, os esforços empreendidos contra a violência não apresentam ainda a eficácia desejada. Um dos motivos têm sido a incipiente utilização do conhecimento a respeito dos complexos fatores que podem tornar as mulheres vulneráveis à opressão masculina como um potente instrumento norteador do processo de trabalho na área da prevenção da violência.

A investigação dos fatores que aumentam a chance de as mulheres sofrerem agressão pelo parceiro oferece informações capazes de subsidiar aos decisores políticos nas escolhas das estratégias e das ações que podem oferecer resultados mais eficientes no combate à violência. Os resultados do estudo poderão subsidiar também os profissionais da saúde, sobretudo os da atenção básica, no reconhecimento de mulheres que estão em risco de sofrer violência conjugal, para, dessa forma, ofertarem ações preventivas.

Nesse contexto, os profissionais, emponderados dos conhecimentos sobre o objeto que pretendem intervir, poderão recorrer a uma rede de apoio às mulheres. Isso requer uma prática multiprofissional e interdisciplinar que, além de ofertar atenção à saúde, ofereça suporte social, jurídico e econômico. Diante do exposto, reitera-se a relevância do tema e do recorte do estudo no contexto atual da saúde, uma vez que essa área ainda necessita de esforços para compreender, reconhecer e intervir adequadamente nos casos de violência conjugal. 


\section{REFERÊNCIAS BIBLIOGRÁFICAS}

1. Ministério da Saúde (BR). Secretaria Especial de Políticas para as Mulheres. Plano Nacional: diálogos sobre violência doméstica e de gênero: construindo políticas públicas. Brasília (DF): MS; 2003 [cited 2011 June 25]. [Internet]. Available from: http://bvsms.saude.gov.br/bvs/ publicacoes/livro.pdf

2. Souza JA, Silva ATMC, Almeida RA, Faustino, WM. Impacto da violência doméstica na saúde mental de mulheres em João Pessoa. In: Colóquio "Violência doméstica sobre mulheres: respostas, dilemas e desafios", 2011 Oct 28; Coimbra, Portugal. Coimbra: Faculdade de Economia da Universidade de Coimbra; 2011. Sala 2.3.

3. Monteiro CFS, Souza IEO. Vivência da violência conjugal: fatos do cotidiano. Texto contexto - enferm [Internet]. 2007[cited 2011 June 25]; 16(1):26-31. Available from: http://www. scielo.br/scielo.php?pid $=$ S0104-07072007000100003\&script $=$ sci arttext

4. Organização Pan-Americana da Saúde (OPAS). Repercussão da Violência na Saúde das Populações Americanas. $44^{\circ}$ Conselho Diretor; 55 Sessão do Comitê Regional. Washington: OPAS; 2003 [cited 2011 June 25]. Available from: http://iris.paho.org/xmlui/bitstream/ handle/123456789/246/cd44-r13-p.pdf?sequence $=4$

5. Organização Pan-Americana da Saúde (OPAS). Relatório Final. $48^{\circ}$ conselho diretor, $60^{\mathrm{a}}$ sessão do comitê regional. Washington: OPAS; 2008 [cited 2011 June 25]. Available from: http:// new.paho.org/hq/dmdocuments/2009/cd48-fr-p.pdf

6. Hosmer DW, Lemeshow S. Applied Logistic Regression. 2nd ed. New York: J.Wiley \& Sons, 2000.

7. Montenegro SG. Modelo de Regressão Logística ordinal em dados categóricos na área da ergonomia experimental. João Pessoa: UFPB, 2009.

8. Triola MF. Introdução à Estatística. 10 ed. Tradução: Vera Regina Lima de Farias e Flores. Rio de Janeiro: LTC, 2008.

9. Sheather SJ. A modern Approach to regression with R. Texas: Springer, 2009.

10. Montgomery DC, Peck EA, Vining GG. Introduction to linear regression analysis. 3 ed. New Delhi: Wiley, 2003.

11. Paula GA. Modelos de Regressão com apoio computacional. São Paulo: USP, 2010.

12. Cordeiro GM, Neto EAL. Modelos Paramétricos. Pernambuco: UFRPE, 2004. 
13. Ryan TP. Modern Regression Methods. 2 ed. New Jersey: Wiley, 2009.

14. Agresti A. Categorical Data Analysis. 2 ed. New Jersey: Wiley, 2002.

15. Hair JFJ, Anderson RE, Tatham RL, Black WC. Análise multivariada de dados. Tradução: Adonai Schlup Sant'Anna Anselmo Chaves Neto. 6 ed. Porto Alegre: Bookman, 2009.

16. Devore JL. Probabilidade e estatística: para engenharia e ciências. Tradução: Joaquim Pinheiro Nunes da Silva. São Paulo: Cengage Learning, 2011.

17. Ministério da Saúde (BR). Conselho Nacional de Saúde. Resolução CNS nº 466, de 12 de dezembro de 2012: diretrizes e normas regulamentadoras de pesquisas envolvendo. Brasília (DF): MS; 2012 [cited 2013 Jul 19]. Available from: http://conselho.saude.gov.br/ultimas noticias/2013/06_jun_14 publicada_resolucao.html

18. Leite FMC, Bravim LR, Lima EFA, Primo CC. Violência contra a mulher: caracterizando a vítima, a agressão e o autor. J. res.: fundam. care. online [Internet]. 2015 [cited 2015 Aug 15]; 7(1):2181-2191. Available from: http://www.seer.unirio.br/index.php/cuidadofundamental/article/ view/3464/pdf 1474

19. Kronbauer JFD, Meneghel SN. Perfil da violência de gênero perpetrada por Companheiro. Rev. Saúde Pública [Internet]. 2005 [cited 2011 June 25]: 39(5):695-701. Available from: http:// www.scielo.br/scielo.php?pid $=\mathrm{S} 0034-89102005000500001 \&$ script $=$ sci_arttext

20. Galvão EF, Andrade SM. Violência contra a mulher: análise de casos atendidos em serviço de atenção à mulher em município do Sul do Brasil. Saúde e Sociedade [Internet]. 2004 [cited 2011 June 25]; 13(2):89-99. Available from: http://www.scielo.br/pdf/sausoc/v13n2/09.pdf

21. Moura MAV, Netto LA, Souza MHN. Perfil sociodemográfico de mulheres em situação de violência assistidas nas delegacias especializadas. Esc Anna Nery [Internet]. 2012 [cited 2015 Aug 15]; 16(3):435 - 442. Available from: http://www.scielo.br/scielo.php?pid=S1414$\underline{81452012000300002 \& \text { script }=\text { sci arttext }}$

22. Stoeckl H, Heise L, Watts C. What puts women at risk for partner violence? Factors associated with violence by a current partner in a nationally representative sample of german women. Injury Prevention [Internet]. 2010 [cited 2011 June 25];16(Suppl 1):244-A245. Available from: http://injuryprevention.bmj.com/content/16/Suppl_1/A244.3.full.pdf+html

23. D’Oliveira FPL, Schraiber LB, França-Junior I, Ludermir AB, Portella AP, Diniz CS, et al. Fatores associados à violência por parceiro íntimo em mulheres brasileiras. Rev. Saúde Pública [Internet]. 2009 [cited 2011 June 25];43(2):299-310. Available from: http://www.scielo.br/scielo. php?pid $=$ S0034-89102009000200011\&script $=$ sci_arttext 
24. Organização Mundial de Saúde (OMS). Estudo multicêntrico da OMS sobre saúde e violência doméstica contra a mulher. Ginebra: OMS; 2005 [cited 2011 June 25]. [Internet] Available from: http://www.who.int/gender/violence/who_multicountry_study/summary_report/ summaryreportSpanishlow.pdf

25. Acosta DF, Gomes VLO, Barlem ELD. Perfil das ocorrências policiais de violência contra a mulher. Acta Paul Enferm[Internet]. 2013 [cited 2015 Aug 15]; 26(6):547-53. Available from: http://www.scielo.br/scielo.php?script=sci_arttext\&pid=S0103-21002013000600007

26. Vieira LJES, Pordeus AMJ, Ferreira RC, Moreira DP, Maia PB, Saviolli KC. Fatores de risco para violência contra a mulher no contexto doméstico e coletivo. Saúde soc [Internet]. 2008 [cited 2011 June 25];17(3):113-125. Available from: http://www.scielo.br/scielo.php?script=sci arttext\&pid=S0104-12902008000300012

27. Murphy E, Ringheim K. Reproductive Health, Gender and Human Rights: A Dialogue. Washington (DC): PATH; 2001 [cited 2011 June 25].[Internet] Available from: http://www.path. org/publications/files/RH-GHR-Dialogue.pdf

28. Monteiro CFS, Araújo TME, Nunes BMVT, Lustosa AR, Bezerra CMJ. A violência contra a mulher atendida em unidade de urgência: uma contribuição da enfermagem. Esc Anna Nery R Enferm [Internet]. 2006 [cited 2011 June 25];10(2):273-9. Available from: http://www.scielo.br/ scielo.php?script $=$ sci_arttext\&pid $=\mathrm{S} 1414-81452006000200015$

29. Schraiber LB, d' Oliveira ANPL, Portella AP, Menicucci E. Violência de gênero no campo da Saúde Coletiva: conquistas e desafios. Ciência \& Saúde Coletiva [Internet]. 2009 [cited 2011 June 25];14(4):1019-1027. Available from: http://www.scielo.br/scielo.php?pid=S1413$\underline{81232009000400009 \& \text { script }=\text { sci arttext }}$

30. Gomes NP, Freire NM. Vivência de violência familiar: homens que violentam suas companheiras. Rev bras enferm [Internet]. 2005 [cited 2011 June 25];58(2):179-179. Available from: http://www.scielo.br/scielo.php?pid=S0034-71672005000200009\&script=sci_arttext

31. Durand JG, Schraiber LB, França-Junior I, Barros C. Repercussão da exposição à violência por parceiro íntimo no comportamento dos filhos. Rev Saúde Pública [Internet]. 2011[cited 2015 Aug 15];45(2):355-64. Available from: http://www.scielo.br/pdf/rsp/v45n2/1940.pdf

32. Butchart A, Phinney A, Check P, Villaveces A. Preventing violence: a guide to implementing the recommendations of the World Report on Violence and Health. Geneva: World Health Organization; 2004 [cited 2011 June 25].[Internet] Available from: http://www.who.int/ violence injury prevention/media/news/08 09 2004/en/

33. Gadoni-Costa LM, Zucatti APN, Dell'Aglio DD. Violência contra a mulher: levantamento 
dos casos atendidos no setor de psicologia de uma delegacia para a mulher. Estudos de Psicologia [Internet]. 2011[cited 2015 Aug 15];28(2): 219-227. Available from: http://www.scielo.br/pdf/ estpsi/v28n2/09.pdf

34. Griebler CN, Borges JL. Violência Contra a Mulher: Perfil dos Envolvidos em Boletins de Ocorrência da Lei Maria da Penha. Psico [Internet]. 2013 [cited 2015 Aug 15]; 44(2): 215 225. Available from: http://revistaseletronicas.pucrs.br/ojs/index.php/revistapsico/article/ view/11463/9640

35. Labronici LM, Ferraz MIR, Trigueiro TH, Fegadoli D. Perfil da violência contra mulheres atendidas na pousada de Maria. Rev Esc Enferm USP [Internet]. 2010 [cited 2015 Aug 15];44 (1): 126-33. Available from: http://www.scielo.br/pdf/reeusp/v44n1/a18v44n1.pdf

36. Sorenson SB, Upchurch DM, Shen H. Violence and Injury in Marital Arguments: Risk Patterns and Gender Differences. American Journal of Public Health [Internet]. 1996 [cited 2011 June 25]; 86(1): 34-40.

37. Xu X, Zhu F, O’Campo P, Koenig MA, Mock V, Campbell J. Prevalence of and Risk Factors for Intimate Partner Violence in China. American Journal of Public Health [Internet]. 2005 [cited 2011 June 25]; 95(1):78-85. Available from: http://www.ncbi.nlm.nih.gov/pmc/ articles/PMC1449856/

38. Heise L, Ellsberg M, Gottemoeller M. Ending Violence Against Women. Population Reports. 1999 Dec; 27 (04): 1-44.

\section{Colaboradores}

Jackeline Abílio de Souza, Ana Tereza de Medeiros, Ulisses Umbelino dos Anjos contribuíram em todas as etapas de produção do artigo: concepção, análise e interpretação dos dados; redação do artigo e revisão crítica do conteúdo intelectual; e aprovação final da versão a ser publicada.

Artigo apresentado em 09/07/15

Artigo aprovado em 18/03/16 Artigo publicado no sistema em 31/03/16 